\title{
ON PILOT CONTAMINATION IN MASSIVE MULTIPLE-INPUT MULTIPLE- OUTPUT SYSTEM WITH LEAST SQUARE METHOD AND ZERO FORCING RECEIVER
}

\author{
Joshi Poojaben Hirenkumar ${ }^{1}$, Jignesh N. Patel ${ }^{2}$ and Hardip K. Shah ${ }^{3}$ \\ ${ }^{1,2}$ Department of Electronics and Communication Engineering, Sardar Vallabhbhai Patel Institute of Technology, India \\ ${ }^{3}$ Department of Electronics and Communication Engineering, Dharmsinh Desai University, India
}

\begin{abstract}
Massive Multiple-Input Multiple-Output (MIMO) wireless communications refers to use of large number of antennas at transmitter and receiver to enhance spectral and energy efficiency. However, its performance is limited by a problem known as pilot contamination. In this paper, we present a comprehensive overview of massive MIMO system and studied degradation in performance due to pilot contamination. To showcase such effects, we have implemented the channel estimation using Least Square (LS) method with random pilots and time shifted pilot scheme through simulations. In this study we have used zero forcing $(\mathrm{ZF})$ receivers. We have also studied performance improvement in presence of pilot using MMSE receiver. Further improvement is achieved in this work by introducing precoding technique for massive MIMO systems.
\end{abstract}

\section{Keywords:}

Massive MIMO, Channel Estimation, Pilot Contamination, MMSE, Precoding, $\mathbf{Z F}$

\section{INTRODUCTION}

MIMO is a multiple antenna technique that allows for transmission of multiple data streams between base station (BS) and user terminals (UTs). The BS with MIMO configuration, typically employs fewer than 10 antennas [1]. A point-to-point MIMO system requires expensive terminals, and the multiplexing gain will disappear at the edges of the cell or in not rich scattering propagation environment [3]. Single user MIMO (SU-MIMO) was adopted to increase spectral efficiency. Multi-user MIMO (MU-MIMO) technology with a maximum of $8 \times 8$ MIMO is recommended in 4G LTE systems. MU-MIMO transmission scheme compared to SU-MIMO provides better spatial multiplexing gains that are effective in improving the average throughput, cell coverage, and reliability of mobile communication systems [1], [2]. Massive MIMO is also known as large-scale antenna systems, very large MIMO, hyper MIMO, full-dimension MIMO, and ARGOS and has been recognized as one of the key technologies in next generation cellular systems.

Massive MIMO is a communication system having a BS with a few hundred antennas simultaneously serve many tens of UTs, equipped with a single antenna, over same time frequency resource [19]. The BS with multiple antennas sends independent data streams to multiple terminals in the same time-frequency resource. Use of massive MIMO is a promising technology that is expected to deliver high data rates as well as enhanced link reliability, coverage, and/or energy efficiency [20].

We have carried out extensive survey on massive MIMO systems and related issues. Pilot contamination is a bottleneck in increasing number of antennas in massive MIMO systems. This problem surface out while channel estimation. To demonstrate the problem of pilot contamination we have implemented different MIMO configuration by gradually increasing number of antennas. For various configurations like $2 \times 2,4 \times 4,16 \times 16$ and $64 \times 64$ antennas, channel estimation is done. In this study we used different kind of pilots like random sequence and time shifted pilots. Through simulation we determined mean square error (MSE) of channel estimation in either case. Since, the channel estimates are necessary for detection, we have measured BER through the simulation with $\mathrm{ZF}$ receiver. The performance improvement is also measured using MMSE receiver. Finally, we added ZF precoder for further improvement in BER.

The rest of this paper is organized as follows: in section 2, we give a potential of massive MIMO system and Drawback of massive MIMO system. Section 3 describes TDD and FDD schemes. In section 4 , We review the different methods for mitigation of Pilot Contamination and brief describe the Time shifted pilot schema. Section 5 gives a Channel estimation using Least Square (LS) method. Then describe the ZF receiver, ZF precoder and MMSE receiver with numerical and simulation results.

\section{THE POTENTIAL OF MASSIVE MIMO}

The basic advantages offered by the features of massive MIMO can be summarized as follows:

- Multiplexing Gain: Aggressive spatial multiplexing used in massive MIMO makes it theoretically possible to increase the capacity 10 times more [4].

- Energy Efficiency: The large antenna arrays can increased energy efficiency in which radiated energy can be concentrated on UE [5]

- Spectral Efficiency: The large number of service antennas in massive MIMO systems and multiplexing to many users to a single user provides the increased spectral-efficiency [5].

- Increased Robustness and Reliability: The large number of antennas allows for more diversity gains that the propagation channel can provide. When the number of antennas increases without bound, data rate and reliability is increased [1], [3].

- Simple Linear Processing: BS antenna is much larger than the UT antenna $(M>>K)$, simplest linear precoders and detectors are optimal [3], [6].

- Cost Reduction in Radio Frequency (RF) Power Components: Due to the reduction in energy consumption, the large array of antennas allows for use of low cost RF amplifiers [1]. 
However, some limitations have been identified. These are: (a) Propagation Models, (b) Hardware Impairment, and (c) Pilot Contamination problem [14]. Although promising, practical implementation of massive MIMO poses a research challenge. In this regard, real world challenges such as channel estimation and pilot design, and propagation effects, theoretical limits in massive MIMO. The Fig. 1 shows the pilot contamination problem in massive MIMO system on channel estimation. It does not vanish even when the number of BS antennas increases. The channel between the transmitter and receiver is estimated from orthogonal pilot sequences which are limited by the coherence time of the channel. The channel estimate at the BS is interfered by UTs of other cells, this effect is called pilot contamination. Pilot contamination decreases the achievable rates in massive MIMO system. Pilots, also referred as training, are known to the receiver and transmitted with the data for channel estimation. But the pilots cannot be orthogonal since they are limited in length.

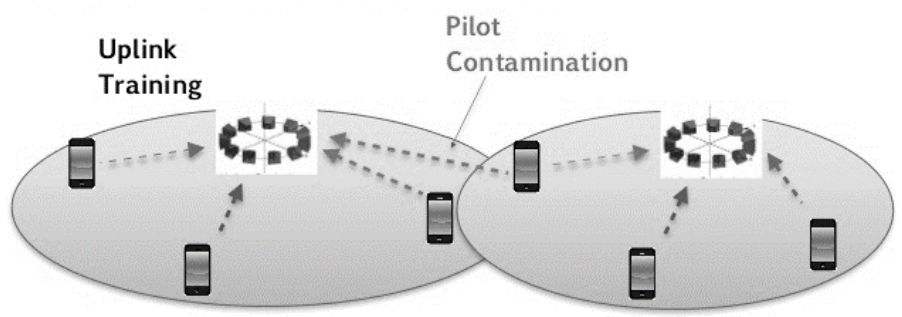

Fig.1. Pilot contamination in massive MIMO system

To achieve the advantages of massive MIMO in practice, each BS needs accurate estimation of the channel state information (CSI), either through feedback or channel reciprocity schemes. CSI plays a key role in a multiuser MIMO system. Forward-link data transmission requires that the base station know the forward channel, and reverse link data transmission requires that the BS know the reverse channel [3].

\section{TDD OR FDD SCHEME}

TDD is considered a better mode to acquire timely CSI in advance wireless systems over FDD because TDD requires estimation, which can be done in one direction and used in both directions; while FDD requires estimation and feedback for both forward and reverse directions, respectively [1], [2], [4]. This paper intends to examine the sources of pilot contamination in massive MIMO systems in the TDD scheme.

In TDD architecture, the use of channel reciprocity and pilots in the UL are key features for its application. Using the notion of reciprocity, it is assumed that the forward channel is equal to the transpose of the reverse channel. Hence, the required channel information is obtained from transmitted pilots on the reverse link from UTs [7]. In a massive MIMO TDD system, the pilot signals which are used to estimate the channels can be contaminated as a result of reuse of non-orthogonal pilot signals in a multi-cell system [10]. This phenomenon causes the inter-cell interference that is proportional to the number of BS antennas [11], which in turn reduces the achievable rates in the network and affect the spectrum efficiency.

The number of pilot symbols for CSI estimation is considered in [7], the minimum number of UL pilot symbols equal to the number of UTs, while in [8], the optimal number of pilot symbols can be larger than the number of antennas if pilot and data power are required to be equal. In the majority of studies carried out on pilot contamination, it is assumed that the same size of pilot signals is used in all cells.

\section{METHODS FOR MITIGATION OF PILOT CONTAMINATION}

In this section, we review existing methods proposed to eliminate the effects of pilot contamination in multi-cell TDD system. The proposed methods have been classified into two categories namely, pilot-based estimation approach and subspacebased estimation approach. In the pilot-based approach, channels of UTs are estimated using orthogonal pilots within the cell and non-orthogonal pilots across the cells, while in the subspacebased estimation approach, the channels of UTs are estimated with or without limited pilots [20].

\subsection{PILOT-BASED ESTIMATION APPROACH}

A time-shifted protocol for pilot transmission was considered, to eliminate pilot contamination in multi-user TDD systems. The transmission of pilot signals in each cell is done by shifting the pilot locations in frames so that users in different cells transmit at non-overlapping times [15]. Although the method looks promising, a major challenge in practice will be the control mechanism needed to dynamically synchronize the pilots across several cells so that they do not overlap. A covariance aided CE method by exploiting the covariance information of both desired and interfering user channels [16]. In this method, a coordinated pilot assignment strategy was proposed based on assigning carefully selected groups of UTs to identical pilot sequences. In this method, Complexity is very high and Do not use under realistic channel conditions. Similar approach [17] A DL training and scheduled UL training scheme in the DL training stage, UTs supported by each BS estimates from the DL pilots of the BS. In the scheduled UL training stage, UTs use the estimated UL pilot symbols. Although this method shows a significant degradation in inter-cell interference and a corresponding increase in UL and DL SINRs, in practice it might be difficult to implement because it requires second order statistics of all the UL channels. In [9], a multi-cell MMSE-based pre-coding scheme, which accounts for the training sequence allocated to all UTs in order to mitigate pilot contamination problem. As a result, pre-coding matrix at each BS is designed to minimize the sum of the mean-square error of signals received at the UTS in the same cell and the mean-square interference occurred at the UTs in other cells. It was shown that the proposed method offers significant performance gains and reduces the inter-cell and intra-cell interference compared to conventional single-cell pre-coding method.

Table.1. Comparison of Pilot based approach

\begin{tabular}{|l|l|}
\hline Pilot based method & \multicolumn{1}{c|}{ Comparison } \\
\hline $\begin{array}{l}\text { Time-shifted pilot } \\
\text { scheme }\end{array}$ & $\begin{array}{l}\text { The Pilot signals in each cell is done by } \\
\text { shifting the pilot locations in frames so } \\
\text { that users in different cells transmit at } \\
\text { non-overlapping times. }\end{array}$ \\
\hline
\end{tabular}




\begin{tabular}{|l|l|}
\hline $\begin{array}{l}\text { Covariance aided } \\
\text { Channel estimation }\end{array}$ & $\begin{array}{l}\text { Complexity is very high, less efficiency } \\
\text { and do not use under realistic channel } \\
\text { conditions }\end{array}$ \\
\hline $\begin{array}{l}\text { DL training and } \\
\text { scheduled UL training } \\
\text { scheme }\end{array}$ & $\begin{array}{l}\text { Used more trainings and difficult to } \\
\text { implement }\end{array}$ \\
\hline MMSE-based scheme & $\begin{array}{l}\text { UTs are the same without differentiating } \\
\text { them based on channels }\end{array}$ \\
\hline
\end{tabular}

In the subspace-based estimation approach, the channels of UTs are estimated with or without limited pilots. The blind method [14] based on subspace approach which is nonlinear channel estimation. The channels can be accurately estimated by decomposition-based method. In this method, the dominant complexity comes from decomposition of the received signal block, which is not often the case in practice.

\subsection{TIME SHIFTED PILOT SCHEME}

Time Shifted protocol shown in Fig.2. It was shown that pilot contamination can be eliminated using the proposed scheme as long as pilots do not overlap in time. The use of power allocation algorithms in combination with the time-shifted protocol in [15] is shown to provide significant gains. With TDD, channel reciprocity is assumed, i.e., the forward link channel vectors are the Hermitian transpose of the reverse link ones. We use reverselink pilots to obtain channel estimates and divide each coherence interval into four phases.

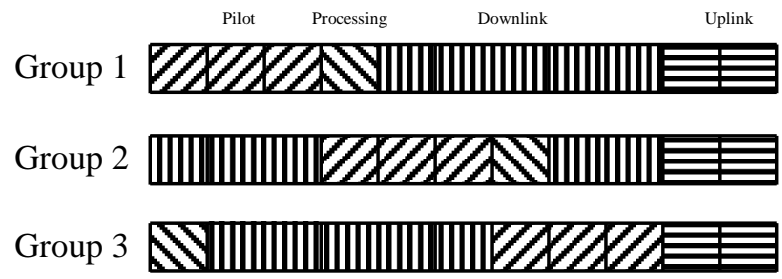

Fig.2. Time shifted pilot scheme

where, $N$ is the number of available pilot sequences at each cell.

- First, each user sends uplink data to its BS for U symbol periods.

- Then, the user sends a pilot sequence of length $\mathrm{N}$ to its BS.

- The BS then uses this pilot to estimate the corresponding channel vector, with which it processes the data received at the uplink phase.

- The BS then transmits downlink data for $D$ symbol periods to its mobile units using the channel estimates as beamforming vectors.

\section{CHANNEL ESTIMATION}

To estimate the channel, we use the pilot signal received from the UTs at the BS. The pilot sequences for different UTs are orthogonal to each other. These properties enable the BS to estimate the channel by eliminating interference between UTs with different pilot sequences during the pilot symbol.

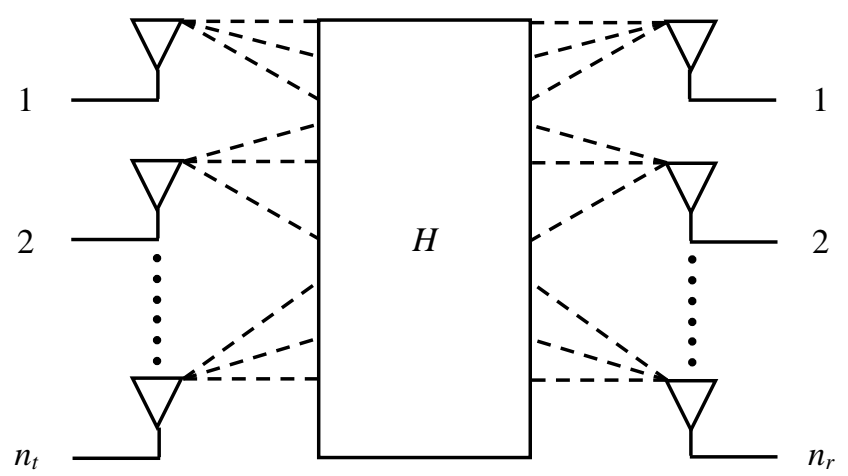

Fig.3. System model of massive MIMO

A massive MIMO system model transmission is given in Fig.3. In this Fig, $n_{t}$ transmit antennas and $n_{r}$ receive antennas are there. The $H$ is channels and the total number of channels is $n_{t} \times n_{r}$. If there are $n_{t}$ transmit antennas and $n_{r}$ receive antennas, then the signals at the receiver can be written as,

$$
Y=X H+V
$$

where, $Y$ is the $n_{r} \times 1$ received matrix, $X$ is the $\mathrm{n}_{t} \times 1$ transmitted matrix, $H$ is the $n_{t} \times n_{r}$ channel matrix, and $V$ is an $n_{r} \times 1$ noise which is AWGN, matrix at the receiver.

If there are $n_{t}$ transmit antennas, $n_{r}$ receive antennas and a pilot length of $N$, then the received signal during pilot transmission can be expressed as,

$$
Y=\Psi H+V
$$

where, $Y$ is the $N \times n_{r}$ received pilot matrix, $\Psi$ is the $N \times n_{t}$ concatenated pilot matrix from $n_{t}$ transmitters, $H$ is the $n_{t} \times n_{r}$ channel matrix and $V$ is an $N \times n_{r}$ noise matrix at the receiver.

A common way to obtain the channel estimate from the received pilot is to use a LS estimate, as follows,

$$
\hat{H}_{L S}=\left(\Psi^{H} \Psi\right)^{-1} \Psi^{H} \mathrm{Y}
$$

where, the value of $\Psi$ is set so that $\Psi^{H} \Psi=I_{n r}$. This can be achieved if pilot sequences from each transmitter are orthogonal to each other and $N \geq n_{t}$. In the case of massive MIMO systems with a large number of BS antennas, but a small number of total UTs antennas, estimation of the uplink channel will be straightforward because the length of uplink pilot should be greater than or equal to the number of total UTs antennas.

To find Mean Square error (MSE) for channel estimation per antenna,

$$
M S E=\left\|\hat{H_{L S}}-H\right\|^{2}
$$

Zero-forcing $(\mathrm{ZF})$ receivers take the inter user interference into account, but neglect the effect of noise. $\mathrm{ZF}$ receiver gives best performance at high SNR. The ZF receiver matrix to recover at receiver,

$$
\Psi^{\wedge}=\left(H^{T} H\right)^{-1} H^{T} Y
$$

To minimize the error of a receiver,

$$
e=\min \left\|Y-H \Psi^{\wedge}\right\|^{\wedge} 2
$$

When receiver is transmitted the signal the transmitter is knows the channel, this techniques is called as precoding technique. Precoding technique also helps to reduce the inter-user interference by focusing the energy on the desired user. ZF precoding techniques in which the inter-user interference can be cancelled out by each user. 
The precoding matrix for ZF precoding can be written as,

$$
P=1 / \beta^{*} H^{H}\left(H H^{T}\right)^{-1}
$$

where,

$$
\beta=[\operatorname{tr}(B B H)]^{0.5}
$$

and,

$$
B=H^{H}\left(H H^{T}\right)^{-1}
$$

The Minimum Mean Square Error (MMSE) receiver aims to reduce the MSE between the estimates the channel and the transmitted signal. It is known that the MMSE receiver maximizes the received SINR. Therefore we can say that MMSE gives best performance at all SNR.

The MMSE receiver matrix to recover at receiver,

$$
\Psi^{\wedge}=\left(H^{T} H+I_{k}\right)^{-1} H^{T} Y
$$

\section{NUMERICAL RESULTS AND DISCUSSIONS}

We have implemented massive MIMO systems with $2 \times 2,4 \times 4$ and $16 \times 16$ antenna configuration. Monte Carlo simulations are carried out with 1600 packets on each SNR. SNR is varied from $1 \mathrm{~dB}$ to $30 \mathrm{~dB}$. BPSK modulation is used. Flat fading channel with Rayleigh distribution is implemented to obtain realistic results.

The Fig.4 shows MSE performance of channel estimation for $2 \times 2,4 \times 4$ and $16 \times 16$ antennas. In this case we have used training matrix of $2 \times 4$, for $4 \times 4$ antennas with training matrix of $4 \times 8$. Similarly, for $16 \times 16$ antennas with training signal is $16 \times 64$.

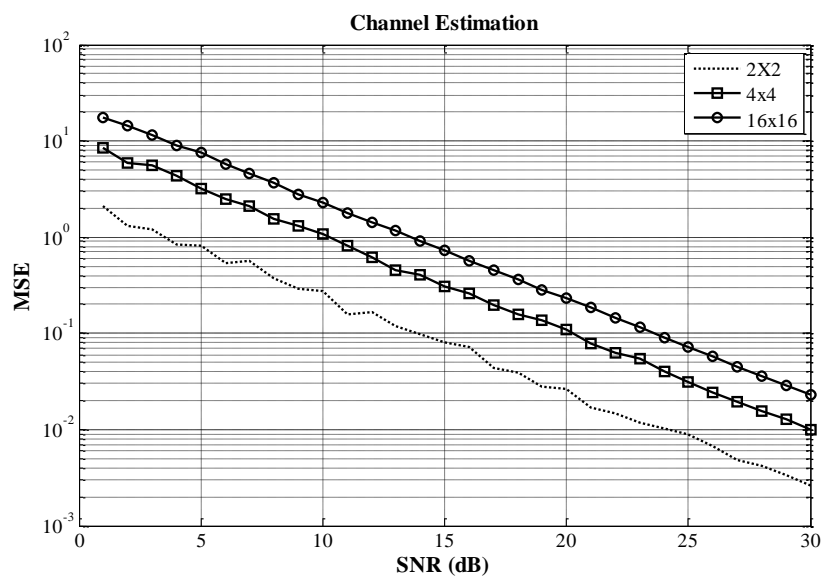

Fig.4. Channel estimation

The graph shows that with increase in SNR the MSE reduces. Performance degradation, even after increasing number of pilots, is observed while increasing number of antennas. It is also observed that at $15 \mathrm{~dB}$ SNR, MSE for $2 \times 2$ is $8 \times 10^{-2}$, for $4 \times 4$ is $2 \times 10^{-1}$ and for $16 \times 6$ is $7 \times 10^{-1}$ as per Fig. 4 . The problem of pilot contamination is demonstrated through simulations. This problem is important because the channel estimates are inevitable for implementation of various detection techniques like ZF or MMSE receivers. Moreover such estimates are required even at transmitter side to implement precoder.

To reduce effect of pilot contamination, we employed time shifted pilot scheme with LS channel estimation. The Fig.5 shows the MSE performance of pilot based time Shifted pilot scheme with channel estimation. The graph shows that when SNR value increases the MSE value reduces. We can see that at $15 \mathrm{~dB}$ SNR, MSE for $2 \times 2$ is $4 \times 10^{-2}$, for $4 \times 4$ is $1 \times 10^{-1}$ and for $16 \times 6$ is $4 \times 10^{-1}$ as per Fig.5. So we can say that MSE value is reduces using time shifted pilot schema. The problem of pilot contamination is degrades using time shifted pilot scheme.

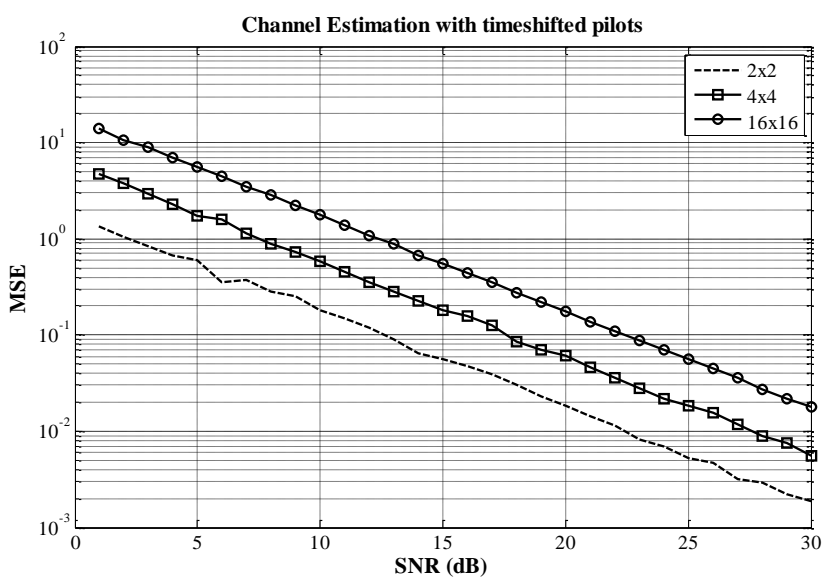

Fig.5. Channel estimation with time shifted pilots

The Fig. 6 shows MSE performance of $16 \times 16$ antennas for channel estimation and channel estimation with time shifted pilot scheme. The graph shows that when SNR value increases the MSE value reduces. We can see that at $15 \mathrm{~dB}$ SNR, for $16 \times 16$ antennas, MSE for channel estimation is $4 \times 10^{-1}$, for channel estimation with time shifted pilots is $7 \times 10^{-1}$ as per Fig.6. So we can say that channel estimation using time shifted pilots are improve the MSE value and pilot contamination problem was degrade.

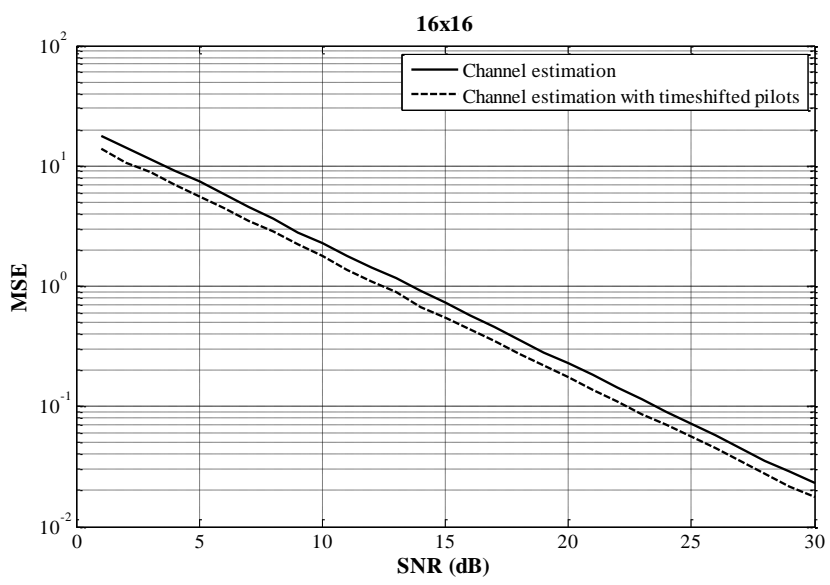

Fig.6. Comparison of channel estimation and channel estimation with time shifted pilots

Here, time shifted pilot scheme is used with LS channel estimation and with the ZF receiver. The Fig.7 shows the BER performance of channel estimation with time shifted pilot using ZF receiver. We have use training matrix of $16 \times 64$ for $16 \times 16$ antennas. Similarly for $64 \times 64$ antennas training matrix is $64 \times 128$. The graph shows that when SNR value increases the BER value reduces. When antennas increases the BER is also increases. We can see that at $15 \mathrm{~dB}$ SNR, for $16 \times 16$ antennas BER is $1 \times 10^{-1}$ for $64 \times 64$ antennas BER is $2 \times 10^{-1}$ as shown in Fig.7. As we have 
noted that for $64 \times 64$ MSE performance is deteriorated as compared to $16 \times 16$, the BER performance is degraded.

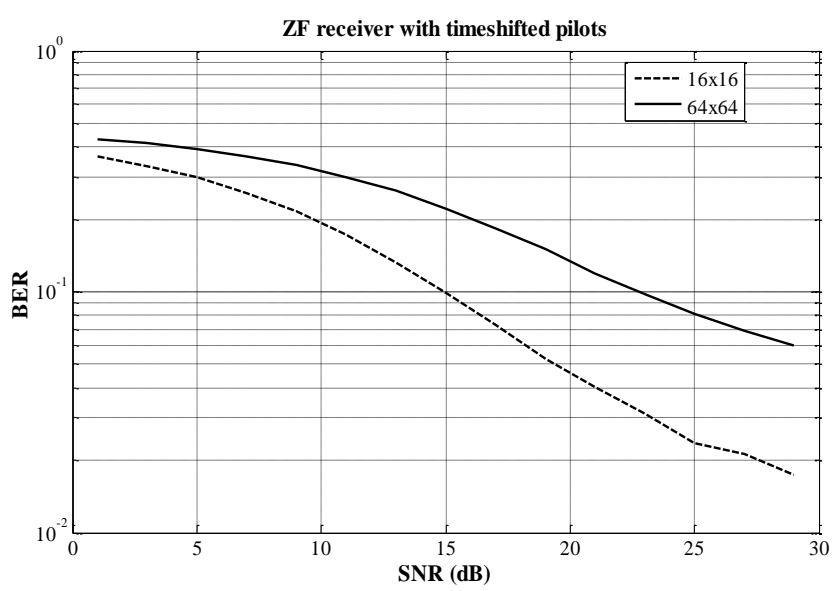

Fig.7. ZF Receiver with time shifted pilots

The time shifted pilot scheme is used with LS Channel estimation with MMSE receiver for $16 \times 16$ MIMO system. The BER performance of this arrangement is shown in Fig.8. The graph shows that we can achieve BER performance of $10^{-3}$ at 20dB SNR. Further increase in SNR cannot reduce the error floor.

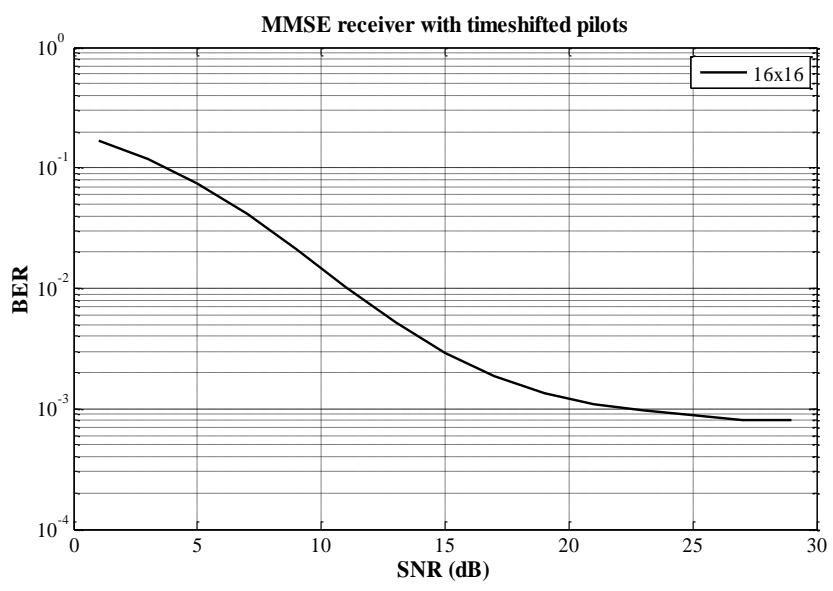

Fig.8. MMSE Receiver with time shifted pilots

The Fig. 9 shows BER performance of $16 \times 16$ antennas for ZF and MMSE receiver with time shifted pilot scheme. The graph shows that when SNR value increases the BER value reduces. We can see that at $15 \mathrm{~dB}$ SNR, for $\mathrm{ZF}$ receiver BER is $1 \times 10^{-1}$, for MMSE receiver BER is $2 \times 10^{-3}$ as shown in Fig.9. So we can say that MMSE gives best results and BER is reduces. The pilot based time shifted pilot scheme is used with LS channel estimation and add the ZF precoder at transmitter side for detection. The Fig.10 shows the BER performance of channel estimation with time shifted pilot using ZF precoder.

The Fig.10 shows that when SNR value increases the BER value reduces. If we compare the $\mathrm{ZF}$ receiver with time shifted pilots for $64 \times 64$ shows in Fig. 7 , so we can say that ZF precoding improve the BER value and pilot contamination problem was degrade with increasing the number of antennas.

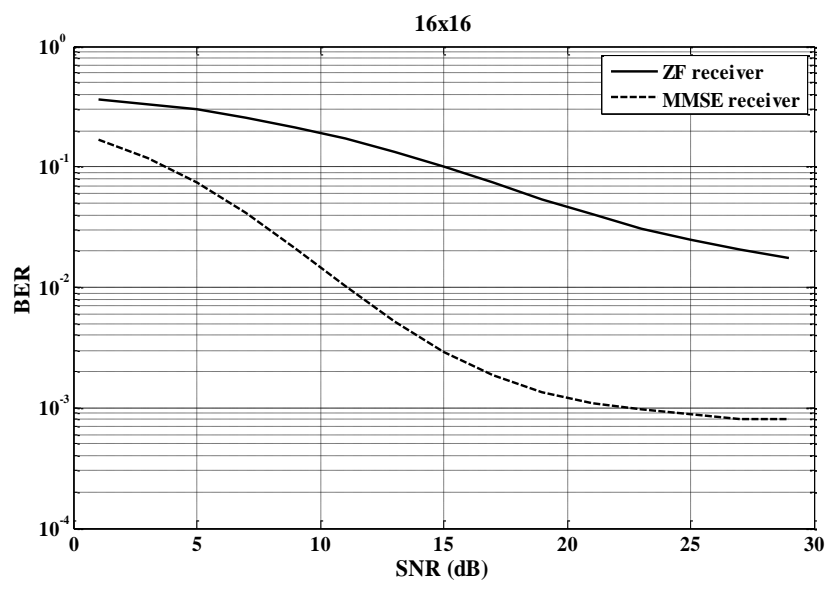

Fig.9. Comparison of ZF and MMSE Receiver for $16 \times 16$

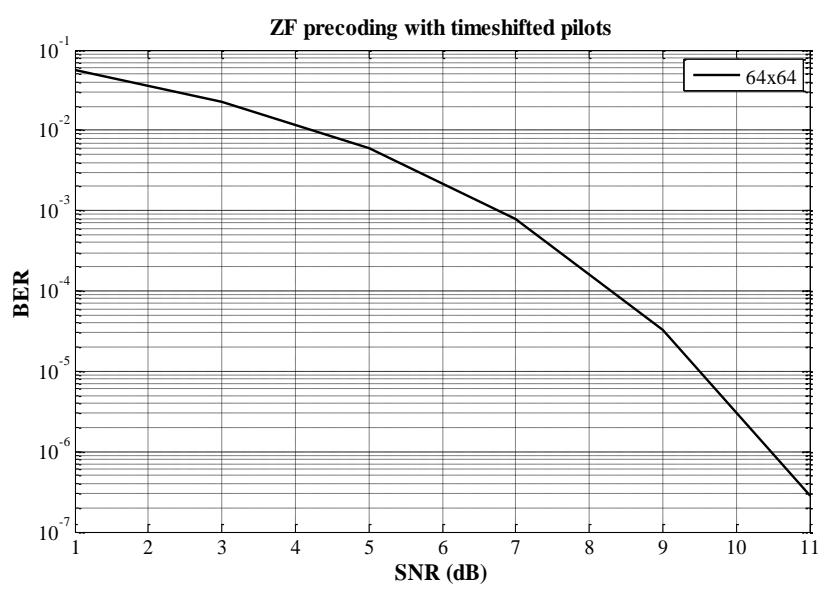

Fig.10. ZF Precoder with time shifted pilots

\section{CONCLUSION}

Comprehensive study of issues related to massive MIMO systems is carried out in this paper. One of the important issue of pilot contamination is studied and its effect is observed for different type of pilots while performing channel estimation. Various receiver techniques are also compared for BER performance. Following inferences derived out of this study.

1. The channel estimation using time shifted pilot scheme reduce the MSE. Hence, impact of pilot contamination can be minimized by using such pilots instead of normal PN sequence pilots.

2. MSE performance of LS channel estimation degrades with increase in number of antennas even after increasing length of pilot sequence. Performance improvement can be achieved using MMSE receiver.

3. ZF precoder improves the BER performance with the requirement of accurate channel estimation.

Finally, we conclude that to minimize pilot contamination we should implement massive MIMO systems with time shifted pilots. For better BER performance, MMSE receiver is preferred over $\mathrm{ZF}$ receiver. If accurate channel estimate is available at transmitter we can go for precoding for improved performance in massive MIMO systems. 


\section{REFERENCES}

[1] F. Rusek, D. Persson, B.K. Lau, E.G. Larsson, T.L. Marzetta, O. Edfors and F. Tufvesson, "Scaling up MIMO: Opportunities and Challenges with Very Large Arrays", IEEE Signal Processing Magazine, Vol. 30, No. 1, pp. 4060, 2013.

[2] L. Lu, G. Li, A. Swindlehurst, A. Ashikhmin and R. Zhang, "An overview of massive MIMO: Benefits and Challenges", IEEE Journal of Selected Topics in Signal Processing, Vol. 8, No. 5, pp. 742-758, 2014.

[3] T.L. Marzetta, "Noncooperative Cellular Wireless with Unlimited numbers of base station Antennas", IEEE Transactions on Wireless Communications, Vol. 9, No. 11, pp. 3590-3600, 2010.

[4] E.G. Larsson, F. Tufvesson, O. Edfors and T.L. Marzetta, "Massive MIMO for Next Generation Wireless Systems", IEEE Communications Magazine, Vol. 52, No. 2, pp. 186195, 2014.

[5] H.Q. Ngo, E.G. Larsson and T.L. Marzetta, "Energy and Spectral Efficiency of very Large Multiuser MIMO Systems", IEEE Transactions on Communications, Vol. 61, No. 4, pp. 1436-1449, 2013.

[6] J. Hoydis, S. Ten Brink and M. Debbah, "Massive MIMO in the UL/DL of Cellular Networks: how many Antennas do we need?", IEEE Journal on Selected Areas in Communications, Vol. 31, No. 2, pp. 160-171, 2013.

[7] Thomas L. Marzetta, "How much training is required for Multiuser MIMO?", Proceedings of Fortieth Asilomar Conference on Signals, Systems and Computers, pp. 359363, 2006.

[8] B. Hassibi and B. Hochwald, "How much training is needed in Multiple Antenna Wireless Links?", IEEE Transactions on Information Theory, Vol. 49, No. 4, pp. 951-963, 2003.

[9] J. Jose, A. Ashikhmin, T.L. Marzetta, and S. Vishwanath, "Pilot Contamination and Precoding in Multi-Cell TDD Systems", IEEE Transactions on Wireless Communications, Vol. 10, No. 8, pp. 2640-2651, 2011.

[10] C. Zhang and G. Zeng, "Pilot Contamination Reduction Scheme in Massive MIMO Multi-Cell TDD Systems", Journal of Computer and System Sciences, Vol. 10, No. 15, pp. 6721-6729, 2014.

[11] J. Jose, A. Ashikhmin, T.L. Marzetta and S. Vishwanath, "Pilot Contamination Problem in Multi-Cell TDD Systems",
Proceedings of IEEE International Symposium on Information Theory, pp. 2184-2188, 2009.

[12] H.Q. Ngo, T.L. Marzetta and E.G. Larsson, "Analysis of the Pilot Contamination Effect in very Large Multicell Multiuser MIMO systems for Physical Channel Models", Proceedings of IEEE International Conference on Acoustics, Speech, and Signal Processing, pp. 3464-3467, 2011.

[13] Qun Yu and Ronglin Li, Guangzhou, "Research on Pilot Pattern Design of Channel Estimation", Journal of Automation and Control Engineering, Vol. 1, No. 2, pp. 160-163, 2013.

[14] R.R. Muller, L. Cottatellucci and M. Vehkaper, "Blind Pilot Decontamination", IEEE Journal of Selected Topics in Signal Processing, Vol. 8, No. 5, pp. 773-786, 2014.

[15] F. Fernandes, A. Ashikhmin and T. Marzetta, "Inter-Cell Interference in Non-cooperative TDD Large Scale Antenna Systems", IEEE Journal on Selected Areas in Communications, Vol. 31, No. 2, pp. 192-201, 2013.

[16] H.F. Yin, D. Gesbert, M. Filippou and Y.Z. Liu, "A Coordinated Approach to Channel Estimation in LargeScale Multiple-Antenna Systems", IEEE Journal on Selected Areas in Communications, Vol. 31, No. 2, pp. 264273, 2013.

[17] J. Zhang, B. Zhang, S. Chen, X. Mu, M. El-Hajjar and L. Hanzo, "Pilot Contamination Elimination for Large-Scale Multiple-Antenna Aided OFDM Systems", IEEE Journal of Selected Topics in Signal Processing, Vol. 8, No. 5, pp. 759772, 2014.

[18] H.Q. Ngo, E.G. Larsson, and T.L. Marzetta, "The Multicell Multiuser MIMO Uplink with very Large Antenna Arrays and A Finite-Dimensional Channel", IEEE Transactions on Communications, Vol. 61, No. 6, pp. 2350-2361, 2013.

[19] Olakunle Elijah, Chee Yen Leow, Abdul Rahman Tharek, Solomon Nunoo and Solomon Zakwoi Iliya, "Mitigating Pilot Contamination in Massive MIMO System-5G", Proceedings of $10^{\text {th }}$ Asian Control Conference, pp. 1-6, 2015.

[20] Olakunle Elijah, Chee Yen Leow, Tharek Abdul Rahman, Solomon Nunoo and Solomon Zakwoi Iliya, "A Comprehensive Survey of Pilot Contamination in Massive MIMO-5G System", IEEE Communications Surveys and Tutorials, Vol. 18, No. 2, pp. 905-923, 2016. 